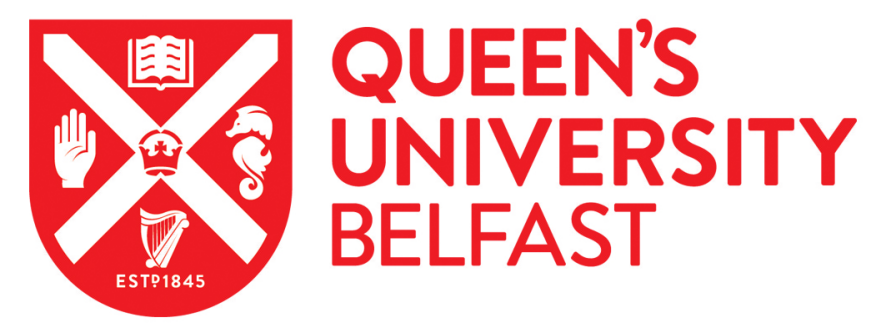

\title{
Ultimate boundedness and robust stabilization of bilinear discrete-time systems
}

\author{
Athanasopoulos, N., Bitsoris, G., \& Vassilaki, M. (2011). Ultimate boundedness and robust stabilization of \\ bilinear discrete-time systems. In Proceedings of the 50th IEEE Conference on Decision and Control and \\ European Control Conference Institute of Electrical and Electronics Engineers Inc.. \\ https://doi.org/10.1109/CDC.2011.6161233
}

Published in:

Proceedings of the 50th IEEE Conference on Decision and Control and European Control Conference

\section{Document Version:}

Peer reviewed version

Queen's University Belfast - Research Portal:

Link to publication record in Queen's University Belfast Research Portal

\footnotetext{
General rights

Copyright for the publications made accessible via the Queen's University Belfast Research Portal is retained by the author(s) and / or other copyright owners and it is a condition of accessing these publications that users recognise and abide by the legal requirements associated with these rights.
}

Take down policy

The Research Portal is Queen's institutional repository that provides access to Queen's research output. Every effort has been made to ensure that content in the Research Portal does not infringe any person's rights, or applicable UK laws. If you discover content in the Research Portal that you believe breaches copyright or violates any law, please contact openaccess@qub.ac.uk. 


\title{
Ultimate boundedness and robust stabilization of bilinear discrete-time systems
}

\author{
Nikolaos Athanasopoulos, George Bitsoris and Marina Vassilaki
}

\begin{abstract}
In this paper nonlinear time-varying and bilinear discrete-time systems with additive bounded disturbances are considered. First, conditions guaranteeing uniform ultimate boundedness for time-varying nonlinear systems are established. Then, algebraic conditions ensuring the existence of polyhedral Lyapunov functions, uniform boundedness and positive invariance for closed-loop bilinear systems are obtained. Finally, these results are applied to various robust stabilization problems for bilinear systems subject to persistent additive disturbances. It is shown that these problems can be reduced to a single or a series of linear programming problems.
\end{abstract}

\section{INTRODUCTION}

The dynamics of most real world systems involve nonlinearities in the state space description as well as time-varying additive terms that represent either uncertainties or persistent external disturbances. For this class of systems, there does not exist any feedback control law ensuring asymptotic stability of an equilibrium state. Instead, a common approach to deal with the stability analysis and control design problem is to identify and characterize subsets of the state space containing the desired equilibrium state with special properties: robust positively invariant sets [1], [2], disturbance invariant sets [3], [4], or ultimately bounded sets [5], [6]. Most of works using these set-theoretic approaches deal with linear systems with polyhedrally bounded disturbances, allowing the systematic computation of approximations of target sets with special properties as minimal robust invariant sets or maximal robust invariant sets.

In this paper, we consider bilinear discrete-time systems with additive disturbances [7]. Stability analysis as well as control design for this class of nonlinear systems still remains a topic of interest, since many processes can naturally be modelled by bilinear systems [8] and bilinear approximations are better than the linear ones, especially when well established identification algorithms can be used [9]. For the case of bilinear systems with no disturbances, a number of control strategies have been proposed, see for example [10], [11], [12]. The problem considered here concerns the determination of a linear state feedback control law ensuring confinement of all closed-loop trajectories starting from an initial states set inside a target set after a finite time. In other words, we search for a feedback control strategy in order to characterize a target subset of the state space with ultimate

N. Athanasopoulos and G. Bitsoris are with the Control Systems Laboratory, Electrical and Computer Engineering Department, University of Patras, Rion 26500, Greece. e-mail: nathanas dece.upatras.gr, bitsorisdece.upatras.gr

M. Vassilaki is with the Department of Electrical Engineering of ASPAITE, Maroussi, Athens-Greece. vassilaki.measpete.gr boundedness. Due to the nonlinearities of the system, it is not possible to achieve global ultimate boundedness, thus another topic of interest is to compute a domain of attraction. As a first step, using the well known comparison systems theory, general conditions guaranteeing uniform ultimate boundedness of nonlinear time-varying systems are established, Then, known conditions related to the existence of polyhedral Lyapunov functions [12] for bilinear systems are modified in order to take into account the additive external disturbances. All these results are used for the development of a systematic robust stabilization methodology which can handle various control objectives.

The paper is organized as follows: In section II, the basic definitions as well as the problem statement are presented. In section III, general conditions ensuring uniform ultimate boundedness for nonlinear time-varying discrete-time systems are established, whereas in section IV these results are modified in order to deal with the class of bilinear systems. In section $\mathrm{V}$, design techniques for various control objectives, are presented along with a numerical example in section VI illustrating the efficacy of these approaches. Conclusions are drawn in section VII.

\section{Problem Statement}

Throughout the paper, capital letters denote real matrices and lower case letters denote column vectors or scalars. $\mathbb{R}^{n}$ denotes the real $n$-space and $\mathbb{R}^{n \times m}$ denotes the set of real $n \times m$ matrices. Given a real $n \times m$ matrix $A=\left(a_{i j}\right)$, $A^{+}=\left(a_{i j}^{+}\right)$and $A^{-}=\left(a_{i j}^{-}\right)$are $n \times m$ matrices with entries defined by the relations $a_{i j}^{+}=\max \left\{a_{i j}, 0\right\}$ and $a_{i j}^{-}=-\min \left\{a_{i j}, 0\right\}$. Thus, $A=A^{+}-A^{-}$. Given a square matrix $D=\left(d_{i j}\right), D^{\delta}=\left(d_{i j}^{\delta}\right)$ denotes the diagonal matrix with $d_{i i}^{\delta}=d_{i i}$ and $D^{\mu}=\left(d_{i j}^{\mu}\right)$ denotes the square matrix with $d_{i i}^{\mu}=0$ and $d_{i j}^{\mu}=d_{i j}$ for $i \neq j$. Thus $D=D^{\delta}+D^{\mu}$. For two $n \times m$ matrices $A=\left(a_{i j}\right)$ and $B=\left(b_{i j}\right)$, $A \odot B=\sum_{i=1}^{n} \sum_{j=1}^{m} a_{i j} b_{i j}$ denotes their component-wise inner product called the Frobenius inner product. The inequality $A \leq B(A<B)$ with $A, B \in \mathbb{R}^{n \times m}$ is equivalent to $a_{i j} \leq b_{i j}\left(a_{i j}<b_{i j}\right)$. Similar notation holds for vectors. Finally, $T$ denotes the time set $T=\{0,1,2, \ldots\}$ and $1_{q}$ denotes the $q \times q$ identity matrix.

Bilinear discrete-time systems are described by difference 
equations of the form

$$
x(t+1)=A x(t)+B u(t)+\left[\begin{array}{c}
x^{T}(t) C_{1} \\
x^{T}(t) C_{2} \\
\vdots \\
x^{T}(t) C_{n}
\end{array}\right] u(t)+E \eta(t)
$$

where $x \in \mathbb{R}^{n}$ is the state vector, $u \in \mathbb{R}^{m}$ is the input vector, $t \in T$ is the time variable and $A \in \mathbb{R}^{n \times n}, B \in \mathbb{R}^{n \times m}$, $C_{i} \in \mathbb{R}^{n \times m}, i=1,2, \ldots, n, E \in \mathbb{R}^{n \times s}$. Function $\eta(t)$, $\eta: T \rightarrow \mathcal{H}$ is assumed to be bounded and represents unknown external disturbances or model uncertainties, $\mathcal{H}$ being a compact subset of $\mathbb{R}^{s}$ containing the origin.

When a linear state-feedback control $u(t)=F x(t)$ with $F \in \mathbb{R}^{m \times n}$ is applied, the resulting closed-loop system is described by a time-varying nonlinear system with second order polynomial nonlinearities:

$$
x(t+1)=(A+B F) x(t)+\left[\begin{array}{c}
x^{T}(t) C_{1} F x(t) \\
x^{T}(t) C_{2} F x(t) \\
\vdots \\
x^{T}(t) C_{n} F x(t)
\end{array}\right]+E \eta(t) .
$$

Due to the unknown disturbances $\eta(t)$, there does not exist any control law rendering the origin an equilibrium state of system (1). For this reason, instead of a desired equilibrium state, a target subset of the state space $\mathbb{R}^{n}$ set containing the origin as an interior point is given. This set is defined by relation $R\left(G_{x}, w_{x}\right) \triangleq\left\{x \in \mathbb{R}^{n}: G_{x} x \leq w_{x}\right\}$ where $G_{x} \in$ $\mathbb{R}^{s \times n}, s \geq n$ and $\max _{1 \leq j \leq s}\left\{\left(G_{x} x\right)_{j}\right\}>0$ for $x \neq 0$ and $w_{x} \in$ $\mathbb{R}^{s}$ is a vector with positive components. Set $R\left(G_{x}, w_{x}\right)$ is a bounded polyhedron containing the origin as an interior point.

The stabilization problem to be investigated is formulated as follows: Given system (1) and a target set $R\left(G_{x}, w_{x}\right)$, determine a linear state-feedback control law $u(t)=F x(t)$ and a set $\Delta, R\left(G_{x}, w_{x}\right) \subset \Delta \subseteq \mathbb{R}^{n}$ so that for any initial state $x\left(t_{0}\right)=x_{0}$ belonging to set $\Delta$ there exists a positive integer $N\left(x_{0}\right)$ such that the corresponding trajectory $x\left(t ; t_{0}, x_{0}\right)$ of the resulting closed-loop system satisfies relation $x\left(t ; t_{0}, x_{0}\right) \in R\left(G_{x}, w_{x}\right)$ for all $t \geq t_{0}+N\left(x_{0}\right)$. In other words, the control problem is to determine a linear statefeedback control law and a domain $\Delta$ so that all trajectories starting from $\Delta$ are transferred to the target set $R\left(G_{x}, w_{x}\right)$ in a finite time and remain in it. Then, the resulting closedloop system is said to be uniformly ultimately bounded in set $R\left(G_{x}, w_{x}\right)$.

Many other variations of relevant control problems may also be considered. Such a control problem consists in the determination of a control law that transfers to the target set $R\left(G_{x}, w_{x}\right)$ all initial states belonging to an a priori given domain of attraction $\Delta$ in a prespecified transfer time $N$.

\section{ULTIMATE BOUNDEDNESS OF NONLINEAR SYSTEMS}

We consider time-varying nonlinear systems described by a difference equation of the form

$$
x(t+1)=f(t, x(t))
$$

where $f: T \times \mathbb{R}^{n} \rightarrow \mathbb{R}^{n}$ is a continuous function. Let $\mathcal{X}$ be a compact subset of the state space $\mathbb{R}^{n}$ containing the origin as an interior point.

Definition 1: System (3) is said to be uniformly ultimately bounded in a subset $\mathcal{X}$ of the state space $\mathbb{R}^{n}$ if there exists a subset $\Delta, \mathcal{X} \subset \Delta \subseteq \mathbb{R}^{n}$ such that for any $t_{0} \in T$ and every initial condition $x\left(t_{0}\right)=x_{0} \in \Delta$ there exists a positive integer $N\left(x_{0}\right)$ such that $x\left(t ; t_{0}, x_{0}\right) \in \mathcal{X}$ for all $t \geq t_{0}+$ $N\left(x_{0}\right)$. Set $\Delta$ is said to be a domain of attraction of set $\mathcal{X}$.

Definition 2: A subset $\mathcal{X}$ of the state space of system (3) is said to be positively invariant if for any $t_{0} \in T$ and every initial condition $x\left(t_{0}\right)=x_{0} \in \mathcal{X}$ the corresponding trajectory remains in $\mathcal{X}$, that is $x\left(t ; t_{0}, x_{0}\right) \in \mathcal{X}$ for all $t \geq t_{0}$.

It is clear that if system (3) is uniformly ultimately bounded in a positively invariant set $\mathcal{X}$, then $\Delta$ is a domain of attraction if and only if for each initial state $x_{0} \in \Delta$ there exists a positive integer $N\left(x_{0}\right)$ such that $x\left(t_{0}+N\left(x_{0}\right) ; t_{0}, x_{0}\right) \in \mathcal{X}$.

The monotone nondecreasing functions defined below play an important role in the development of the results of this paper:

Definition 3. A vector valued function $h(t, y), h: T \times$ $\mathbb{R}^{q} \rightarrow \mathbb{R}^{q}$ is said to be monotone nondecreasing if for any $t \in T$ all its components $h_{i}\left(t, y_{1}, y_{1}, \ldots y_{q}\right) \quad i=1,2, \ldots, q$ are nondecreasing with respect to $y_{j} j=1,2, \ldots, q$.

If function $h(t, y)$ is monotone nondecreasing, then system

$$
y(t+1)=h(t, y(t))
$$

is monotone, in the sense that $y_{0} \leq \hat{y}_{0}$ implies $y\left(t ; t_{0}, y_{0}\right) \leq$ $y\left(t ; t_{0}, \hat{y}_{0}\right)$ for all $t_{0} \in T$ and $t \geq t_{0}$.

In the following lemma, conditions for the uniform ultimate boundedness of monotone systems (4) are established:

Lemma 1. If function $h(t, y), h: T \times \mathbb{R}^{q} \rightarrow \mathbb{R}^{q}$ is monotone nondecreasing and there exist positive real numbers $r_{1}$, $r_{2}$, and $\varepsilon<1$ such that

$$
h(t, r w) \leq \varepsilon r w \quad \forall r \in\left[r_{1}, r_{2}\right], \quad \forall t \in T
$$

then system (4) is uniformly ultimately bounded in set

$$
R\left(1_{q}, r_{1} w\right)=\left\{y \in \mathbb{R}^{q}: y \leq r_{1} w\right\},
$$

and

$$
R\left(1_{q}, r_{2} w\right)=\left\{y \in \mathbb{R}^{q}: y \leq r_{2} w\right\}
$$

is a domain of attraction.

Proof: From (5) it follows that for any $t_{0} \in T$

$$
y\left(t_{0}+1 ; t_{0}, r_{2} w\right)=h\left(t_{0}, r_{2} w\right) \leq \varepsilon r_{2} w
$$

for any $t_{0} \in T$. Let $N$ be the minimal positive integer satisfying inequality $\varepsilon^{N} r_{2}<r_{1}$. Such a $N$ exists because $\varepsilon<1$. Then $r_{1} \leq \varepsilon^{k} r_{2} \leq r_{2} \quad \forall k=1,2, \ldots, N-1$ and taking into account (5) and the hypothesis that function $h(t, y)$ is monotone nondecreasing, by induction, it can be easily proven that

$$
y\left(t_{0}+k ; t_{0}, r_{2} w\right) \leq \varepsilon^{k} r_{2} w \quad \forall k=1,2, \ldots, N-1
$$


and

$$
\begin{aligned}
y\left(t_{0}+N ; t_{0}, r_{2} w\right) & =h\left(t_{0}+N-1, y\left(t_{0}+N-1 ; t_{0}, r_{2} w\right)\right) \\
& \leq h\left(t_{0}+N-1, \varepsilon^{N-1} r_{2} w\right) \leq \\
& \leq \varepsilon^{N} r_{2} w<r_{1} w .
\end{aligned}
$$

On the other hand, inequality $h\left(t, r_{1} w\right) \leq r_{1} w, \forall t \in T$, implies the positive invariance of set $R\left(1_{q}, r_{1} w\right)$ w.r.t. to the monotone system (4) [13]. Therefore,

$$
y\left(t_{0}+k ; t_{0}, r_{2} w\right) \leq r_{1} w \quad \forall t \geq t_{0}+N .
$$

Finally, since system (4) is monotone, if $y_{0} \leq r_{1} w$, that is if $y_{0} \in R\left(1_{q}, r_{1} w\right)$, then $y\left(t ; t_{0}, y_{0}\right) \leq y\left(t ; t_{0}, r_{1} w\right)<r_{1} w$, or equivalently $y\left(t ; t_{0}, y_{0}\right) \in R\left(1_{q}, r_{1} w\right)$, for all $t_{0} \in T$ and $t \geq t_{0}+N$ Consequently, system (4) is uniformly ultimately bounded in $R\left(1_{q}, r_{1} w\right)$ and $R\left(1_{q}, r_{2} w\right)$ is a domain of attraction.

We are now in a position to establish conditions guaranteeing the uniform ultimate boundedness of system (3) in a compact subset $\mathcal{X}$ of the state space $\mathbb{R}^{n}$. Let $P(v, w)$ be the subset of the state space $\mathbb{R}^{n}$ defined by relation

$$
P(v, w) \triangleq\left\{x \in \mathbb{R}^{n}: v(x) \leq w\right\}
$$

where $v(x), v: \mathbb{R}^{n} \rightarrow \mathbb{R}^{q}$ is a continuous vector valued function such that $v(0)=0$.

Theorem 1. If for a continuous function $v(x), v: \mathbb{R}^{n} \rightarrow$ $\mathbb{R}^{q}$ there exist a monotone nondecreasing function $h(t, y), h$ : $T \times \mathbb{R}^{q} \rightarrow \mathbb{R}^{q}$ and positive real numbers $r_{1}, r_{2}$, and $\varepsilon<1$ such that

$$
\begin{gathered}
P\left(v, r_{1} w\right) \subseteq \mathcal{X} \subset P\left(v, r_{2} w\right) \\
v[f(t, x(t)] \leq h(t, v(x)) \\
h(t, r w) \leq r \varepsilon w \quad \forall r \in\left[r_{1}, r_{2}\right], \quad \forall t \in T
\end{gathered}
$$

then system (3) is uniformly ultimately bounded in set $\mathcal{X}$ and $P\left(v, r_{2} w\right)$ is a domain of attraction of set $\mathcal{X}$.

Proof: Since function $h(t, y)$ is monotone nondecreasing, if $v\left(x_{0}\right)=y_{0}$ then $v\left[x\left(t ; t_{0}, x_{0}\right)\right] \leq y\left(t ; t_{0}, y_{0}\right)$ for all $t_{0} \in T$ and $t \geq t_{0}, y\left(t ; t_{0}, y_{0}\right)$ being the trajectory of the comparison system

$$
y(t+1)=h(t, y(t))
$$

with initial condition $y\left(t_{0}\right)=y_{0}$. If $x_{0} \in P\left(v, r_{2} w\right)$, that is if $v\left(x_{0}\right) \leq r_{2} w$ then $v\left[x\left(t ; t_{0}, x_{0}\right)\right] \leq y\left(t ; t_{0}, r_{2} w\right)$ for all $t \geq t_{0}$. By virtue of Lemma 1 this implies inequality

$$
v\left[x\left(t ; t_{0}, x_{0}\right)\right] \leq y\left(t ; t_{0}, r_{2} w\right) \leq \varepsilon^{N} r_{2} w \leq r_{1} w
$$

for all $t \geq t_{0}+N$, that is $x\left(t ; t_{0}, x_{0}\right) \in P\left(v, r_{1} w\right) \subseteq \mathcal{X}$ for all $t \geq t_{0}+N$.

Remark 1. From (8) it follows that, under the hypotheses of this theorem, all initial states $x_{0}$ belonging to the domain of attraction $P\left(v, r_{2} w\right)$ are transferred to the target set $\mathcal{X}$ in a time not exceeding the minimal positive integer $N$ that satisfies inequality

$$
\varepsilon^{N} \leq \frac{r_{1}}{r_{2}}
$$

Remark 2. It is known [13] that the existence of a nondecreasing function $h(t, y)$ satisfying (6) and inequality $h(t, w) \leq w \quad \forall t \in T$ is a necessary and sufficient condition for the positive invariance of set $P(v, w)$ w.r.t. system (3). Therefore, hypotheses of this theorem, besides uniform ultimate stability in set $\mathcal{X}$, imply the positive invariance of all sets $P(v, r w) \forall r \in\left[r_{1}, r_{2}\right]$.

We shall use this result to establish conditions of robust stability for nonlinear systems in the presence of additive persistent disturbances. Systems of this class are described by state equations of the form

$$
x(t+1)=f(t, x(t))+\eta(t)
$$

where $f: T \times \mathbb{R}^{n} \rightarrow \mathbb{R}^{n}$ with $f(t, 0) \equiv 0$ and $\eta: T \rightarrow \mathcal{H}$, $\mathcal{H}$ being a compact subset of $\mathbb{R}^{n}$. Function $\eta(t)$ represents model uncertainties or/and external persistent disturbances.

Theorem 2. If for a continuous function $v(x), v: \mathbb{R}^{n} \rightarrow$ $\mathbb{R}^{q}$ there exist a monotone nondecreasing function $h(t, y), h$ : $T \times \mathbb{R}^{q} \rightarrow \mathbb{R}^{q}$ and positive real numbers $r_{1}, r_{2}$, and $\varepsilon<1$ such that

$$
\begin{gathered}
P\left(v, r_{1} w\right) \subseteq \mathcal{X} \subset P\left(v, r_{2} w\right) \\
v[f(t, x)] \leq h(t, v(x)) \\
h(t, r w)+d \leq r \varepsilon w \quad \forall r \in\left[r_{1}, r_{2}\right], \quad \forall t \in T .
\end{gathered}
$$

where $d \in \mathbb{R}^{q}$,

$$
d_{i}=\max _{t \in T}\left\{v_{i}(\eta(t))\right\} \quad i=1,2, \ldots, q
$$

then system (10) is uniformly ultimately bounded in set $\mathcal{X}$ and $P\left(v, r_{2} w\right)$ is a domain of attraction of set $\mathcal{X}$.

\section{STABILIZATION OF BILINEAR SYSTEMS}

The result established in Theorem 2 can be used for the design of robust controllers for various types of nonlinear systems subject to additive persistent disturbances. In this section we develop a systematic method for stabilization of bilinear systems described by state equations (1).

We select function $v(x)$ and vector $w$ to be

$$
v(x)=G^{*} x=\left[\begin{array}{c}
G \\
-G
\end{array}\right] x, \quad w=\left[\begin{array}{l}
w_{1} \\
w_{2}
\end{array}\right]
$$

where $G \in \mathbb{R}^{p \times n}, \operatorname{rank} G=n$ and $w_{i} \in \mathbb{R}^{p} \quad i=1,2$ are vectors with positive components. Then set $P(v, w)=$ $Q\left(G, w_{1}, w_{2}\right)$ where

$$
Q\left(G, w_{1}, w_{2}\right) \triangleq\left\{x \in \mathbb{R}^{n}:-w_{2} \leq G x \leq w_{1}\right\}
$$

is a bounded polyhedron containing the origin as an interior point. Let $y_{1}=\left[\begin{array}{llll}y_{11} & y_{12} & \ldots & y_{1 p}\end{array}\right]^{T}=(G x)^{T}, y_{2}=$ $\left[\begin{array}{llll}y_{21} & y_{22} & \cdots & y_{2 p}\end{array}\right]^{T}=-(G x)^{T}$ and $Y^{M}=\left(y_{i j}^{M}\right)$, $Y^{m}=\left(y_{i j}^{m}\right) \quad$ be $p \times p$ matrices whose elements are defined by the relations $y_{i j}^{M} \triangleq \max \left(y_{1 i} y_{1 j}, y_{2 i} y_{2 j}\right), \quad y_{i j}^{m} \triangleq$ $\max \left(y_{1 i} y_{2 j}, y_{2 i} y_{1 j}\right)$. Then we can prove [12] that for the closed-loop system (2) and function $v(x)$ defined by (14) 
condition (12) of Theorem 2 is satisfied by the nondecreasing function

$h(y)=\left[\begin{array}{cc}H^{+} & H^{-} \\ H^{-} & H^{+}\end{array}\right]\left[\begin{array}{l}y_{1} \\ y_{2}\end{array}\right]+\left[\begin{array}{c}D_{1}^{+} \odot Y^{M}+D_{1}^{\mu^{-}} \odot Y^{m} \\ \vdots \\ D_{p}^{+} \odot Y^{M}+D_{p}^{\mu^{-}} \odot Y^{m} \\ D_{1}^{-} \odot Y^{M}+D_{1}^{\mu^{+}} \odot Y^{m} \\ \vdots \\ D_{p}^{-} \odot Y^{M}+D_{p}^{\mu^{+}} \odot Y^{m}\end{array}\right]$ and

where $H \in \mathbb{R}^{p \times p}$ and $D_{j} \in \mathbb{R}^{p \times p} j=1,2, \ldots, p$ are matrices satisfying relations

$$
\begin{gathered}
G(A+B F)=H G \\
\sum_{i=1}^{n} g_{j i} C_{i} F=G^{T} D_{j} G \quad j=1,2, \ldots, p .
\end{gathered}
$$

Let also $d \in \mathbb{R}^{2 p}$ be the vector defined by relation

$$
d_{i}=\left\{\begin{array}{cc}
\max _{t \in T}\left\{(G E \eta(t))_{i}\right\} & i=1,2, \ldots, p \\
-\min _{t \in T}\left\{(G E \eta(t))_{i}\right\} & i=p+1, p+2, \ldots, 2 p
\end{array}\right.
$$

Then, by applying the result stated in Theorem 2 we establish the following conditions of uniform ultimate boundedness of closed-loop system (2) in set $R\left(G_{x}, w_{x}\right)$.

Theorem 3. If for a matrix $G \in \mathbb{R}^{p \times n}, \operatorname{rank} G=n$ and a vector $w \in \mathbb{R}^{p}, w>0$, there exist matrices $H \in \mathbb{R}^{p \times p}$ and $D_{j} \in \mathbb{R}^{p \times p} j=1,2, \ldots, p$ and positive real numbers $r_{1}, r_{2}$ and $\varepsilon<1$ satisfying relations (16)-(17) and such that

$$
Q\left(G, r_{1} w_{1}, r_{1} w_{2}\right) \subseteq R\left(G_{x}, w_{x}\right) \subset Q\left(G, r_{2} w_{1}, r_{2} w_{2}\right)
$$

and

$$
h(r w)+d \leq r \varepsilon w \quad \forall r \in\left[r_{1}, r_{2}\right],
$$

with function $h(y)$ and vector $d$ defined by (15) and (18) respectively, then the closed-loop system (2) is uniformly ultimately bounded in $R\left(G_{x}, w_{x}\right)$ and $Q\left(G, r_{2} w_{1}, r_{2} w_{2}\right)$ is a domain of attraction

This result cannot be applied in this form to the design of robust controllers for bilinear system (1). For this reason, we first replace set conditions (19) by equivalent algebraic ones. By applying Farkas Lemma, set conditions (19) are equivalent to the existence of nonnegative matrices $K \in$ $\mathbb{R}^{s \times 2 p}$ and $L \in \mathbb{R}^{2 p \times s}$ such that

$$
\begin{gathered}
K G^{*}=G_{x} \\
r_{1} K w \leq w_{x} \\
L G_{x}=G^{*} \\
L w_{x}<r_{2} w .
\end{gathered}
$$

Conditions (21)-(22) which are equivalent to set relation $Q\left(G, r_{1} w_{1}, r_{1} w_{2}\right) \subseteq R\left(G_{x}, w_{x}\right)$ can also be replaced by the linear inequalities

$$
G_{x} x^{(j)} \leq w_{x} \quad j=1,2, \ldots, 2 p
$$

where $x^{(j)}$ denote the vertices of polyhedron $Q\left(G, r_{1} w_{1}, r_{1} w_{2}\right)$.

Next, we replace (20) by an equivalent condition non depending on variable $r$ but only on the bounds $r_{1}$ and $r_{2}$. Let $H^{*} y$ and $g^{*}(y)$ be the linear and the nonlinear part respectively of function $h(y)$, namely

$$
\begin{gathered}
H^{*} y=\left[\begin{array}{cc}
H^{+} & H^{-} \\
H^{-} & H^{+}
\end{array}\right] y \\
g^{*}(y)=\left[\begin{array}{c}
D_{1}^{+} \odot Y^{M}+D_{1}^{\mu^{-}} \odot Y^{m} \\
\vdots \\
D_{p}^{+} \odot Y^{M}+D_{p}^{\mu^{-}} \odot Y^{m} \\
D_{1}^{-} \odot Y^{M}+D_{1}^{\mu^{+}} \odot Y^{m} \\
\vdots \\
D_{p}^{-} \odot Y^{M}+D_{p}^{\mu^{+}} \odot Y^{m}
\end{array}\right]
\end{gathered}
$$

Then, condition (20) is written as

$$
\begin{array}{cc}
H^{*} r w+g^{*}(r w)+d \leq r \varepsilon w & \forall r \in\left[r_{1}, r_{2}\right], \\
r H^{*} w+r^{2} g^{*}(w)+d \leq r \varepsilon w & \forall r \in\left[r_{1}, r_{2}\right] .
\end{array}
$$

Functions

$$
r\left[\left(H^{*}-\varepsilon 1_{2 p}\right) w\right]_{j}+r^{2} g_{j}^{*}(w)+d_{j} \quad j=1,2, \ldots, 2 p
$$

are convex. Therefore if

$$
r_{i}\left[\left(H^{*}-\varepsilon 1_{2 p}\right) w\right]_{j}+r_{i}^{2} g_{j}^{*}(w)+d_{j} \leq 0 \quad j=1,2, \ldots, 2 p
$$

for $i=1,2$ then

$$
r\left[\left(H^{*}-\varepsilon 1_{2 p}\right) w\right]_{j}+r^{2} g_{j}^{*}(w)+d_{j} \leq 0 \quad j=1,2, \ldots, 2 p
$$

for all $r \in\left[r_{1}, r_{2}\right]$ or, equivalently,

$$
r H^{*} w+r^{2} g^{*}(w)+d \leq r \varepsilon w \quad \forall r \in\left[r_{1}, r_{2}\right] .
$$

Consequently, condition (20) is equivalent to conditions

$$
r_{i} H^{*} w+r_{i}^{2} g^{*}(w)+d \leq r_{i} \varepsilon w \quad i=1,2 .
$$

Thus, we establish the following result:

Theorem 4. If for a matrix $G \in \mathbb{R}^{p \times n}, \operatorname{rank} G=n$ and vectors $w_{i} \in \mathbb{R}^{p}, w_{i}>0 \quad i=1,2$, there exist matrices $H \in \mathbb{R}^{p \times p}, D_{j} \in \mathbb{R}^{p \times p} j=1,2, \ldots, p, K \in \mathbb{R}^{s \times 2 p}$, $K \geq 0, L \in \mathbb{R}^{2 p \times s}, L \geq 0$ and positive real numbers $r_{1}, r_{2}$ and $\varepsilon<1$ satisfying relations (16)-(17) and such that

$$
\begin{gathered}
K G^{*}=G_{x} \\
r_{1} K w \leq w_{x} \\
L G_{x}=G^{*} \\
L w_{x}<r_{2} w \\
r_{i} H^{*} w+r_{i}^{2} g^{*}(w)+d \leq r_{i} \varepsilon w \quad i=1,2
\end{gathered}
$$

with matrix $H^{*}$ and function $g^{*}(y)$ defined by (26) and (27) respectively, then the closed-loop system (2) is uniformly ultimately bounded in set $R\left(G_{x}, w_{x}\right)$ and $Q\left(G, r_{2} w_{1}, r_{2} w_{2}\right)$ is a domain of attraction. 


\section{Design TEChNiQues}

Many different approaches to the stabilization of bilinear systems can be established by applying the results stated in Theorem 4. In this section, we develop systematic design methods that reduce the robust stabilization problem to one or a sequence of linear programming problems.

According to Theorem 4, a linear control law $u=F x$ is a solution to the control problem if for a matrix $G \in \mathbb{R}^{p \times n}$ $\operatorname{rank} G=n$ and positive vectors $w_{1}, w_{2} \in \mathbb{R}^{p}$, there exist matrices $H \in \mathbb{R}^{p \times p}, D_{j} \in \mathbb{R}^{p \times p} j=1,2, \ldots, p, K, L$ and positive real numbers $r_{1}, r_{2}$ and $\varepsilon<1$ satisfying relations

$$
\begin{gathered}
G(A+B F)=H G \\
\sum_{i=1}^{n} g_{j i} C_{i} F=G^{T} D_{j} G \quad j=1,2, \ldots, p \\
r_{1} H^{*} w+r_{i}^{2} g^{*}(w)+d \leq r_{i} \varepsilon w, i=1,2 \\
K G^{*}=G_{x} \\
r_{1} K w \leq w_{x} \\
L G_{x}=G^{*} \\
L w_{x} \leq r_{2} w \\
K, L \geq 0
\end{gathered}
$$

For the determination of a solution to the control problem we first compute $r_{1}$ so that conditions (33) and (34) guaranteeing that $Q\left(G, r_{1} w_{1}, r_{1} w_{2}\right) \subseteq R\left(G_{x}, w_{x}\right)$ are satisfied. This can be done by solving the linear programming problem linear programming problem

$$
\min _{K, r_{1}^{-1}}\left\{r_{1}^{-1}\right\}
$$

under constraints

$$
\begin{gathered}
K G^{*}=G_{x} \\
K w \leq r_{1}^{-1} w_{x} \\
K \geq 0 .
\end{gathered}
$$

Then a control law $u(t)=F x(t)$ making system (1) uniformly ultimately bounded in $R\left(G_{x}, w_{x}\right)$ and $Q\left(G, r_{2} w_{1}, r_{2} w_{2}\right)$ a domain of attraction can be determined by solving the linear programming problem

$$
\min _{F, H, D_{1}, \ldots, D_{p}, \varepsilon}\{\varepsilon\}
$$

under constraints (30)-(32). The computed control $u=F x$ is a solution to the control problem if the optimal nonnegative value $\varepsilon_{\text {opt }}$ of parameter $\varepsilon$ is less than 1 .

Minimization of parameter $\varepsilon$ results to a faster transient behavior because from (9) it follows that all initial states belonging to the domain of attraction $Q\left(G, r_{2} w_{1}, r_{2} w_{2}\right)$ are transferred to the target set $R\left(G_{x}, w_{x}\right)$ in a time not exceeding $N$, where

$$
N=\frac{1}{\log \left(\varepsilon_{o p t}\right)} \log \left(\frac{r_{1}}{r_{2}}\right) .
$$

Next we consider the control problem with guaranteed performance. In this case, parameter $\varepsilon$ that characterizes the transient behavior of the closed-loop system is a priori given. Then the problem is the determination of a control law $u=F x$ and of a domain of attraction $Q\left(G, r_{2} w_{1}, r_{2} w_{2}\right)$ so that all initial states belonging to this set are transferred to the target set $R\left(G_{x}, w_{x}\right)$ in a time $N^{*}$. Such a control law and a domain of attraction can be determined by solving the optimization problem

$$
\max _{F, H, D_{1}, \ldots, D_{p}, L, r_{2}}\left\{r_{2}\right\}
$$

with constraints (30)-(32), (35), (36) $L \geq 0$ and $\varepsilon=\varepsilon^{*}$ where

$$
\log \left(\varepsilon^{*}\right)=\frac{1}{N^{*}} \log \left(\frac{r_{1}}{r_{2}}\right) .
$$

It can be easily shown that this optimization problem is convex and is equivalent to a series of linear programming problems.

\section{NUMERICAL EXAMPLE}

We consider a second order bilinear system (1) with system matrices $A=\left[\begin{array}{cc}1 & 0.01 \\ 0.02 & 0.8\end{array}\right], \quad B=\left[\begin{array}{l}0.09 \\ 0.09\end{array}\right]$, $C_{1}=\left[\begin{array}{c}0.001 \\ 0\end{array}\right], \quad C_{2}=\left[\begin{array}{c}0 \\ -0.004\end{array}\right], \quad E=I_{2}$. The system is subject to bounded additive disturbances $\eta(t) \in \mathcal{H}$, where $\mathcal{H}=R\left(G_{\eta}, w_{\eta}\right)=\left\{\eta \in \mathbb{R}^{2}: G_{\eta} \eta \leq w_{\eta}\right\}$, with $G_{\eta}=\left[\begin{array}{cc}0.96 & -0.22 \\ -0.87 & 0.49 \\ 0.24 & 0.97\end{array}\right], \quad w_{\eta}=\left[\begin{array}{l}0.019 \\ 0.004 \\ 0.030\end{array}\right]$. It is also given a target set $R\left(G_{x}, w_{x}\right)=\left\{x \in \mathbb{R}^{2}: G_{x} x \leq w_{x}\right\}$ and a desired domain of attraction $Q\left(G, r_{2} w, r_{2} w\right)=\{x \in$

$\left.\mathbb{R}^{2}:-r_{2} w \leq G x \leq r_{2} w\right\}$ where $G_{x}=\left[\begin{array}{cc}1 & 0.1 \\ -0.4 & 1 \\ -1 & -0.1 \\ 0.4 & -1\end{array}\right]$,
$w_{x}=\left[\begin{array}{l}0.34 \\ 0.51 \\ 0.34 \\ 0.51\end{array}\right], G=\left[\begin{array}{ll}1 & 0 \\ 0 & 1\end{array}\right], w=\left[\begin{array}{l}0.3 \\ 0.3\end{array}\right], \quad r_{2}=5$.
The first problem to be solved is the determination of a linear state feedback control law $u(t)=K x(t)$ so that all trajectories starting from set $Q\left(G, r_{2} w, r_{2} w\right)$ are transferred to the interior of the target set $R\left(G_{x}, w_{x}\right)=\left\{x \in \mathbb{R}^{2}\right.$ : $\left.G_{x} x \leq w_{x}\right\}$ in finite time $N$ and remain in it.

In order to determine a value of parameter $r_{1}$ such that $Q\left(G, r_{1} w, r_{1} w\right) \subseteq R\left(G_{x}, w_{x}\right)$ we solve the linear programming problem described in (38)-(41). The optimal value of $r_{1}^{-1}$ is equal to 1 . Therefore, with $r_{1}=1$ we obtain $Q(G, w, w) \subseteq R\left(G_{x}, w_{x}\right)$.

Then a control law rendering the system uniformly ultimately bounded in set $R\left(G_{x}, w_{x}\right)$ with set $Q(G, 5 w, 5 w)$ being a domain of attraction is obtained by solving the linear programming problem $\min _{F, H, D_{1}, \ldots, D_{r}, \varepsilon}\{\varepsilon\}$ under constraints (30)-(32) and $r_{1}=1, r_{2}=5$. The optimal value of parameter $\varepsilon$ has found to be $\varepsilon_{\text {opt }}=0.99$ where $F=$ $\left[\begin{array}{ll}-1.0405-0.1111\end{array}\right]$. Since $\varepsilon<1$, the state-feedback 


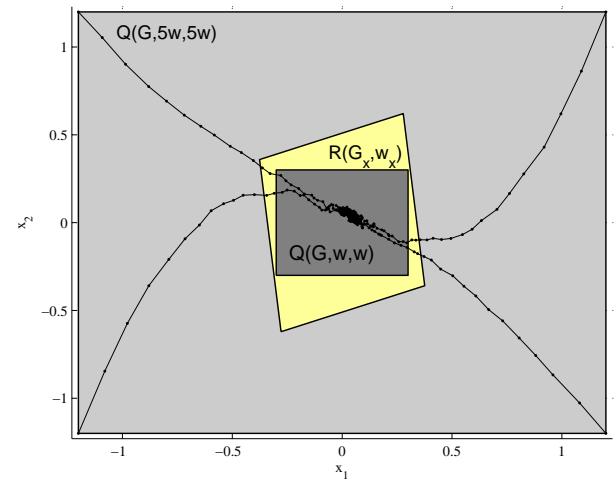

Fig. 1. The target set $R\left(G_{x}, w_{x}\right)$, the domain of attraction $Q(G, 5 w, 5 w)$, the positively invariant $\operatorname{set} Q(G, w, w)$ and trajectories of the closed-loop system starting from the vertices of $Q(G, 5 w, 5 w)$.

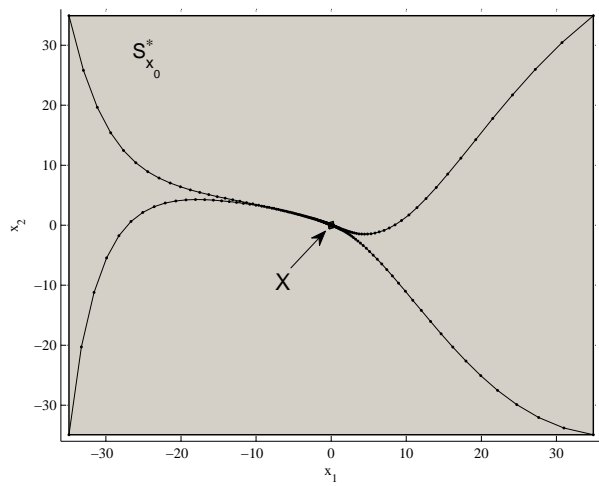

Fig. 2. The maximized domain of attraction $Q\left(G, r_{2}^{*} w, r_{2}^{*} w\right)$ and the trajectories of the closed-loop system starting from the vertices of this set.

control $u(t)=-1.0405 x_{1}(t)-0.1111 x_{2}(t)$ is a solution to this control problem. The closed-loop bilinear system trajectories starting from the vertices of set $Q(G, 5 w, 5 w)$ are shown in Fig. 1, along with the domain of attraction $Q(G, 5 w, 5 w)$, the target set $S\left(G_{x}, w_{x}\right)$ and the positively invariant set $Q(G, w, w)$.

The second problem to be solved concerns the computation of the maximal domain of attraction $Q(G, r w, r w)$. Since we are interested to find a large initial condition set, we select a slow rate of convergence $\varepsilon=0.9999$. Solving the optimization problem $\max _{F, H, D_{1}, \ldots, D_{r}, r_{2}}\left\{r_{2}\right\}$ under constraints(30)-(32), $r_{1}=1$ and $\varepsilon=0.9999$, the optimal value of $r_{2}$ and the corresponding gain matrix has been found to be $r_{2}^{*}=116.4$ where $F=\left[\begin{array}{ll}-0.9092 & -0.1111\end{array}\right]$. It should be noticed that this optimization problem is convex and it can be reduced to a sequence of linear programming problems. In Fig. 2, the maximized domain of attraction $S\left(G, r_{2}^{*} w, r_{2}^{*} w\right)$ for the closed-loop system is shown together with the trajectories emanating from the vertices of the set.

\section{CONCLUSIONS}

A new approach to the robust stabilization of discrete time bilinear systems with additive external disturbances has been presented. First, the necessary theoretical background, originating from comparison systems theory and leading to conditions guaranteeing uniform ultimate boundedness for nonlinear time varying systems has been established. Then, using polyhedral Lyapunov functions, algebraic conditions for the uniform ultimate boundedness of bilinear systems subject to external persistent disturbances have been developed. These conditions have been used for the development of systematic methods for the design of robust linear controllers. Thus, the computation of stabilizing linear state feedback control laws is reduced to a single or a series of linear programming problems. It should be noticed that the proposed robust stabilization methodology is versatile because many different design objectives can be considered, and can be naturally extended to the constrained stabilization case.

\section{REFERENCES}

[1] S.V. Rakovic, E.C. Kerrigan, K.I. Kouramas, and D.Q. Mayne, "Invariant approximations of the minimal robust positively invariant set", IEEE Transactions on Automatic Control, vol. 50, pp.406-410, 2005.

[2] S.R. Rakovic, and K.I. Kouramas, "Invariant approximations of the minimal robust positively invariant set via finite time Aumann integrals", in Proceedings of the 46th IEEE conference on Decision and Control, New Orleans, USA, pp.194-199, 2007.

[3] I. Kolmanovsky, and E.G. Gilbert, "Theory and computation of disturbance invariant sets for discrete-time linear systems", Mathematical Problems in Engineering, vol. 4, pp. 317-367, 1998.

[4] C.J. Ong, and E.G. Gilbert, "The minimal disturbance invariant set: Outer approximations via its partial sums", Automatica, vol. 42, pp.1563-1568, 2006.

[5] M.J. Corless, and G. Leitmann, "Continuous state feedback guaranteeing uniform ultimate boundedness for uncertain dynamic systems", IEEE Transactions on Automatic Control, vol. AC-26, pp.1139-1144, 1981

[6] F. Blanchini, "Ultimate boundedness control for uncertain discretetime systems via set-induced Lyapunov functions", IEEE Transactions on Automatic Control, vol. 39, pp. 428-433, 1994.

[7] C. Bruni, G.D. Pillo, and G. Koch, "Bilinear systems: An appealing class of nearly linear systems in theory and applications", IEEE Transactions on Automatic Control, vol. AC-19, 334-348, 1974.

[8] R.R. Mohler, and W.J. Kolodziej, "An overview of bilinear systems theory and applications", IEEE Transactions on Systems, Man and Cybernetics, SMC-10, 683-688, 1980.

[9] W. Favoreel, B. De Moor, and P. Van Overschee, (1999), "Subspace identification of bilinear systems subject to white inputs", IEEE Transactions to Automatic Control, vol. 44, 1157-1165, 1999.

[10] W. Liao, M. Cannon, and B. Kouvaritakis, "Constrained MPC using feedback linearization on SISO bilinear systems with unstable inverse dynamics", International Journal of Control, vol. 78, 638-646, 2005.

[11] A. B. Fontes, C.E.T. Dorea, and M.R.S. Garcia, "An iterative algorithm for constrained MPC with stability of bilinear systems", in Proceedings of the 16th Mediterranean Conference on Control and Automation, Ajaccio, France, 25-27 June, pp. 1526-1531, 2008.

[12] N. Athanasopoulos, and G. Bitsoris, "Unconstrained and constrained stabilization of bilinear discrete-time systems using polyhedral Lyapunov functions", International Journal of Control, vol. 13, pp.24832493, 2010.

[13] G. Bitsoris, and E. Gravalou, "Comparison principle, positive invariance and constrained regulation of nonlinear systems", Automatica, 31, 217-222, 1995. 